\title{
Optimization of Cleaning Management Improves the Efficiency of the Continuous Surgery
}

\author{
Haiyan Li*, Zhiqiang Su, Shanghai Ma, Zhiling Ou \\ Operating Room of the First Affiliated Hospital of Jinan University, Guangzhou, China \\ Email address: \\ zengtianyu26@126.com (Haiyan Li) \\ ${ }^{*}$ Corresponding author
}

To cite this article:

Haiyan Li, Zhiqiang Su, Shanghai Ma, Zhiling Ou. Optimization of Cleaning Management Improves the Efficiency of the Continuous Surgery. American Journal of Nursing Science. Vol. 9, No. 3, 2020, pp. 107-111. doi: 10.11648/j.ajns.20200903.14

Received: March 17, 2020; Accepted: April 1, 2020; Published: April 13, 2020

\begin{abstract}
Objective: To explore optimized processes and experience of cleaning operating room during continuous surgery. Method: Selected surgery cases from January to December 2018 randomly, 300 cases were using traditional methods of cleaning and 300 cases with improved methods of cleaning, we compared and analyzed the cleaning time and cleaning effect between two different methods, and investigated the changes of action ability of cleaning team, and analyzed the changes of doctor-patient satisfaction. Results: In 2018, we used improved cleaning management methods, cleaning time, cleaning effect, cleaning team action and patient or doctor satisfaction are significantly better than the traditional cleaning methods. Conclusion: In operating room, scientific cleaning management can significantly improve the efficiency of the operation, prevent the spread of bacteria in the operating room, improve the efficiency of the operating room, and thereby increase surgeon and patient satisfaction.
\end{abstract}

Keywords: Continuous Surgery, Operation Room Efficiency, Cleaning Management

\section{Background}

The operating room is an important medical resource of the hospital, which is also an important source of hospital income and expenditures [1]. Surgical requirements are increasing, and the operating room space is limited. Surgical operations require a table distribution to complete the operation [2]. The surgical procedure is tedious and time-consuming, it requires the cooperation of the surgical department, anesthesia department and operating room [3]. One of the key points is that the cleaning process of the operating room is ignored. In order to make reasonable use of the operating room resources [4], we began to continuously optimize the cleaning process of the surgical table distribution in February 2018, reduce the surgical table distribution time, and achieve good results, as described below.

\section{Materials and Methods}

\subsection{Clinical Data}

Our hospital is a large-scale third-level general hospital with 20 clean laminar flow operating rooms, and the volume of annual operation is about 20,000. Since February 2018, the management reform of the cleaning operation has been carried out, the cleaning process has been transformed and fully promoted in the operating room. Selected 300 patients (traditional cleaning method) including 162 males and 138 females which were from February to December 2017 undergoing table surgery were included in the control group randomly. Age 23 to 78 years. Selected 300 patients (improved cleaning method) from February to December 2018 who underwent surgery were included in the observation group, including 158 males and 142 females. Age 17 to 77 years. There was no significant difference in the age, gender, and educational level between the two groups of surgical patients $(P>0.05)$, and they were comparable.

\subsection{Cleaning Methods}

Traditional cleaning method: The roving nurse or surgeon will notify the cleaning staff to perform cleaning by verbal notification after the operation. The cleaning tools are traditional mop and bucket. The cleaning staff cleans the area without regular training and without fixed responsibility.

Improved cleaning methods: A. Notification process: The main nurse in the operating room notices the cleaning staff 
through real-time walkie-talkie to perform cleaning when the surgery is about to end; B. Cleaning tools: use removable floor mop instead of traditional Non-removable mop and bucket, one operation, one mop, disinfection, cleaning and drying. C. Dividing the operation area and optimizing the cleaning staff scheduling: dividing three operating areas according to the distribution of the operating room to ensure the shortest path to the cleaning operating room; increasing the work of mobile cleaning staff according to the peak time of the receiving operation, and flexible scheduling according to the receiving operation situation. D. Formulate and improve the management system of cleaning personnel: including job responsibilities, safe operating procedures, disinfection and isolation systems, infection management systems, reward and punishment methods, and evaluation systems, etc., and set up full-time personnel to supervise and inspect and implement monthly regular training for cleaning personnel., Monthly assessment of the quality of cleaning work.

Table 1. Comparison of traditional cleaning and improved cleaning.

\begin{tabular}{lll}
\hline Group & Traditional cleaning & Improved cleaning \\
\hline Notification process & Surgeon or visiting nurse notice Cleaners & Operating room nurse watching surveillance video to notify cleaners \\
Notification device & None & Real-time walkie-talkie \\
Cleaning tools & Traditional mop & Removable machine washable floor mop \\
Area of responsibility & Random & Fixed point \\
Cleaners shift & Hospital shift schedule & Schedule by time \\
Training and assessment & None & Regular \\
\hline
\end{tabular}

\subsection{Observation Indicators and Analysis Methods}

Analyzing the cleaning time of the two cleaning methods after the operation is from the end of the first operation to the completion of cleaning. Observe the cleaning time used by the traditional cleaning method and the improved cleaning method, and compare the types of bacteria carried by the removable mop and the traditional mop. Quantity, comparative analysis of cleaning quality. Among them, the operating room cleaning management team members fill in the self-assessment questionnaire scores, at the same time record the satisfaction of the patients and surgeons to the operation.

\subsection{Statistical Methods}

SPSS19.0 statistical software was used for statistical analysis. The t-test was used for comparison between the two groups. $P<0.05$ was considered statistically significant.

\section{Results}

\subsection{Improved Cleaning Methods Significantly Speed up Cleaning}

The "cleaner cleaning efficiency questionnaire" was designed to investigate on-site cleaning time, selected 300 sets of traditional cleaning methods and 300 sets of modified cleaning methods randomly, and collected the results. The average cleaning time for traditional cleaning methods for table-top surgery: $(35 \pm 4.28) \mathrm{min}$, of which the average time for the cleaning staff to reach the operating room after the operation is: $(15 \pm 2.28) \mathrm{min}$, the average time for cleaning in the operating room: $(20 \pm 3.28) \mathrm{min}$; The average cleaning time for the improved cleaning method to handle the table surgery: $(12 \pm 2.14) \mathrm{min}$, in which the average time for the cleaning staff to reach the operating room after the operation is: $(2 \pm 0.23) \mathrm{min}$, the average time for cleaning in the operating room: $(10 \pm 1.21) \mathrm{min}$. The results were analyzed by t-test, * indicates $P<0.05$. The results show that the improved cleaning method significantly speeds up cleaning.

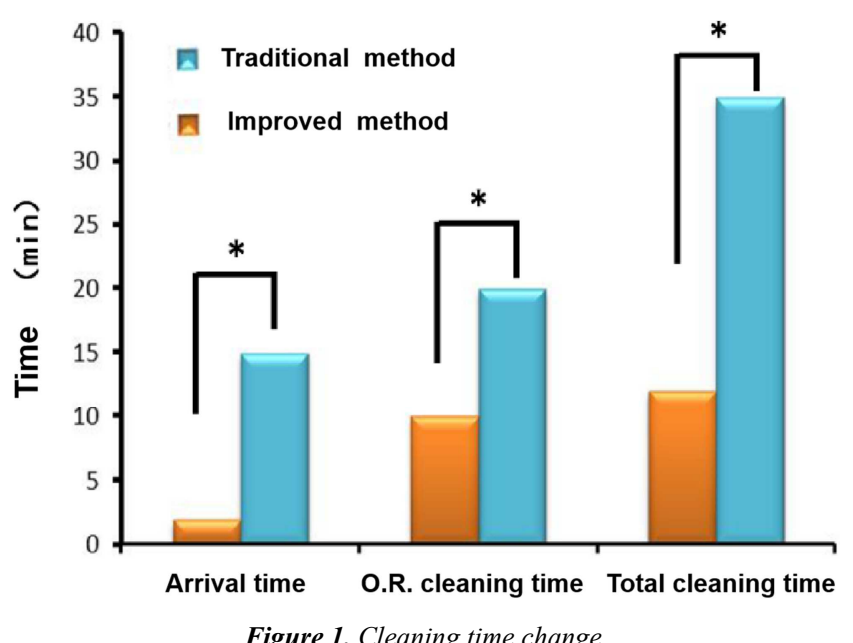

\subsection{Improved Cleaning Methods to Reduce Bacterial Contamination}

Some studies have reported that traditional mop moist mops carry a large number and variety of colonies after cleaning and disinfection, which can make the bacteria move and spread, are prone to nosocomial infections [5]. Operating room cleaners are an important part of controlling nosocomial infections. They are the specific implementers of cutting off the transmission route of the source of the disease. The traditional colonies and new cleaning methods were used to measure the colonies and numbers carried by mops before cleaning. The numbers of traditional mops were Staphylococcus epidermidis (126 \pm 13.59$) \mathrm{cfu} / \mathrm{cm}^{2}$, Staphylococcus aureus (45 \pm 5.19$)$, and hemolytic streptococci $(65 \pm 6.39) \mathrm{cfu} / \mathrm{cm}^{2}$ and E. coli $(75 \pm 8.1) \mathrm{cfu} / \mathrm{cm}^{2}$; the number of new ground drag bacteria is Staphylococcus epidermidis $(4 \pm 0.59) \mathrm{cfu} / \mathrm{cm}^{2}$, Staphylococcus aureus $(2 \pm$ $0.13)$, hemolytic chain Cocci $(3 \pm 0.23) \mathrm{cfu} / \mathrm{cm}^{2}$ and E. coli $(8$ $\pm 1.2) \mathrm{cfu} / \mathrm{cm}^{2}$. Two groups of mop cultures were performed 
30 times, and each culture was performed independently. The results were analyzed by t-test, $*$ indicates $P<0.05$. The new machine-washable floor mop is used to improve cleaning efficiency and ensure cleaning quality.

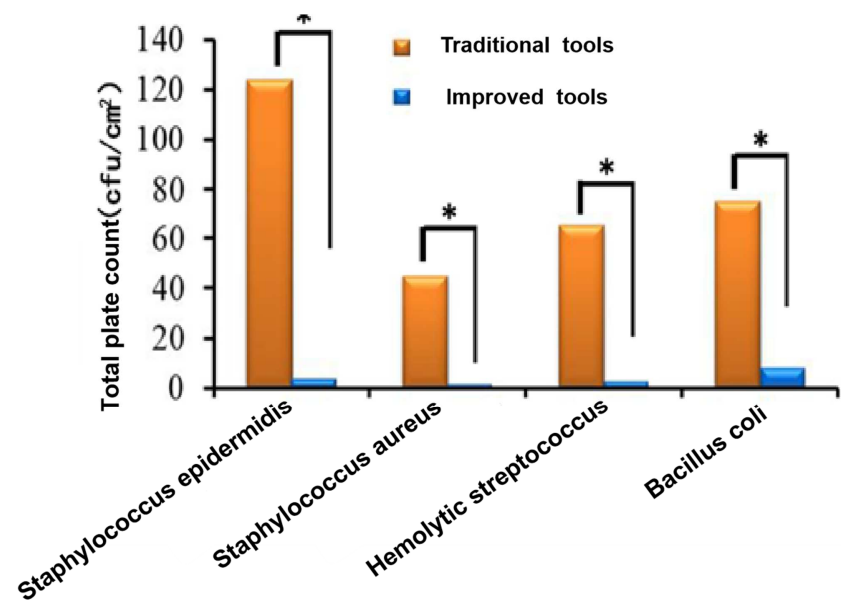

Figure 2. Evaluation of cleaning effect.

\subsection{Improved Cleaning and Enhanced Cleaning Team Ability}

The 10 team members of the cleaning management team of the operating room improved their self-scoring before and after cleaning improvement, including responsibility, self-confidence, enthusiasm, problem solving ability and team cohesion. Score before cleaning improvement: responsibility $(34 \pm 5.45)$, self-confidence $(40 \pm 3.21)$, enthusiasm ( $32 \pm 2.10)$, problem-solving ability ( $40 \pm 5.39)$, and team cohesive responsibility $(36 \pm 4.51)$. The score after cleaning improvement: responsibility (76 \pm 7.36), self-confidence $(66 \pm 5.43)$, motivation $(88 \pm 9.37)$, problem-solving ability $(80 \pm 9.24)$, and team cohesive responsibility $(84 \pm 7.61)$. The results were analyzed by t-test, * indicates $P<0.05$. The results showed that after the improved cleaning management, the cleaning team members' ability scores were significantly improved compared with before the improvement.

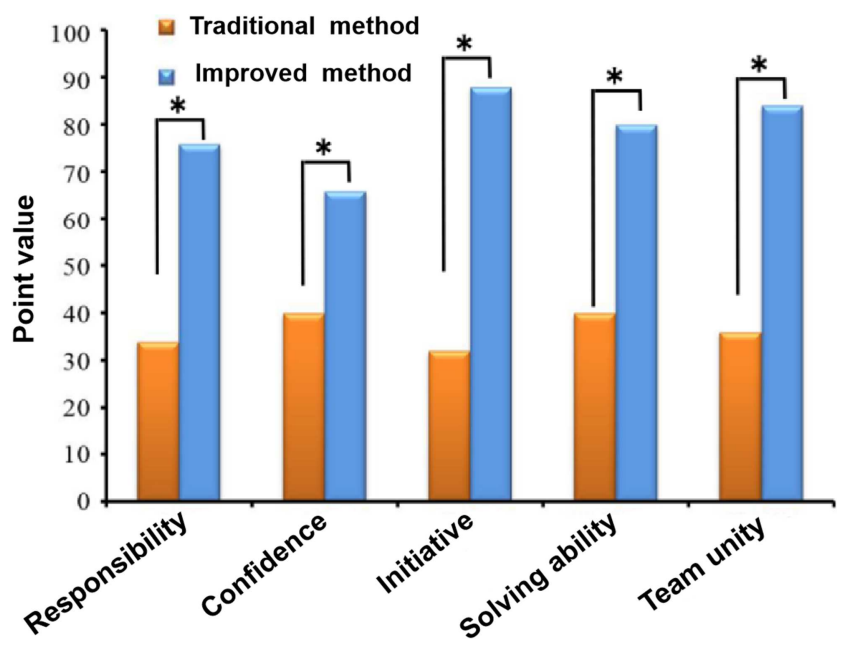

Figure 3. Cleaning team ability score.

\subsection{Improved Cleaning and Increased Doctor-Patient Satisfaction}

A total of 300 patients and doctors were selected randomly before and after the improvement of cleaning management to conduct a satisfaction survey and scored, with a perfect score of $100 \%$. Doctor and patient satisfaction before cleaning management improvement were $(74 \% \pm 6.56 \%)$ and $(81 \% \pm$ 5.74), and doctor and patient satisfaction after cleaning management improvement were: $(90 \% \pm 7.92 \%)$ and $(92 \%) \pm$ $6.85)$. The results were analyzed by paired t test. * Indicates $P$ $<0.05$. The results are statistically significant. After the improvement of cleaning management, the satisfaction of doctors and patients is significantly improved.

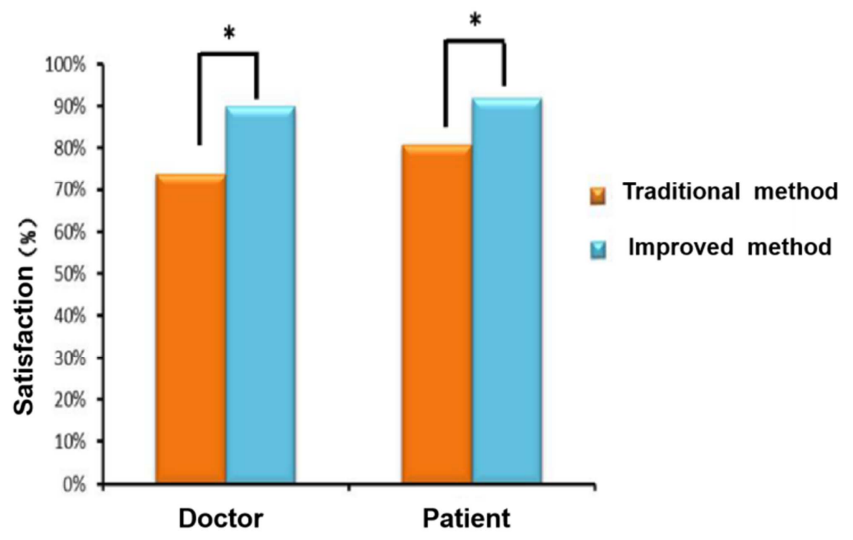

Figure 4. Doctor and Patient Satisfaction Survey.

\section{Discussion}

\subsection{Necessity of Optimizing Cleaning Management in the Operating Room}

The traditional management of the operating room gets rare attention on the management of the surgical process, resulting in low working efficiency of the staff in various departments related to surgery; resulting in long working hours and high intensity of the nurses, anesthesiologists and surgeons in the operating room [6]. In particular, the low efficiency of the table operation leads to the extension of the operating room ineffective time, causing a high and waste cost of operating room [7]. The uncertainty of the operation time of the docking table often causes some contradictions and hidden dangers [8-10]. One of the key links affecting the operation time of the table surgery is the cleaning efficiency of the operating room, and the cleaning management is easy for everyone to ignore. The operating room managers urgently need to re-integrate and optimize the cleaning process, revise the corresponding management system, improve all aspects of work and operating efficiency.

\subsection{Optimizing Cleaning Management and Improving Operating Room Utilization}

Improving the cleaning efficiency of table-top surgery is first and foremost to improve the management system of cleaning personnel in clean operating rooms. Formulating the 
duties of cleaning personnel, safe operating procedures, sterilization and isolation systems, infection management systems, reward and punishment methods, evaluation systems, set up full-time personnel to supervise and implement $[11,12]$. We divide the cleaning operation area of the cleaning staff, and notify the cleaning staff via walkie-talkie as soon as possible to ensure the shortest route to the cleaning operation room; optimize the cleaning staff scheduling, and increase the work of mobile security staff according to the peak time of the operation. In order to make reasonable use of the operating room resources, we have begun to continuously optimize the cleaning process of the surgical table. First of all, the operating room uses removable mop, replacing the traditional non-removable mop and bucket, updating the cleaning tools and optimizing the notification method (Table 1), which can shorten the average time by 13 minutes. The operating room can save 10 minutes compared to the traditional dragging, and the average operation can save 23 minutes (Figure 1), which greatly improves the efficiency of the surgical table.

\subsection{Improving Cleaning Management to Reduce Bacterial Contamination}

To improve cleaning efficiency and ensure cleaning quality, implement a procedure of one operation one place mopping cloth, disinfection, cleaning and drying standby. To achieve WST/367-2012 "Technical Specifications for Disinfection of Medical Institutions" and meet the requirements of laminar operating room environmental hygiene, prevent cross infection from occurring. After implementing two different methods to mop and wipe the ground, various bacteria including staphylococcus epidermidis, staphylococcus aureus, streptococcus hemolyticus, and E. coli were significantly reduced (Figure 2). It can be seen that by rationally managing the mop in the operating room in detail, the disinfection management of the floor in the operating room is strengthened, and the occurrence of nosocomial infection can be better controlled.

\subsection{Improving Cleaning Management, Operating Ability and Doctor-Patient Satisfaction of the Operating Room Cleaning Management Team}

The operating room is a key department of the hospital. The turnover of surgical patients depends on whether the operating room can operate effectively. The speed of the operation is one of the signs that reflects the efficient operation of the operating room [13]. The reasons for the excessive length of the operation are as follows: uncertain preparation time before surgery, significantly longer patient transfer time, long cleaning time in the operating room, improper sequence of surgical arrangements for patients with special infections, and delays in the time for the surgeon to attend the stage $[14,15]$. Among them, the cleaning time of the operating room is a key factor that restricts the speed of the operation. Some studies have found that the long waiting time for the surgeries can easily increase the patient's fear and cause anxiety [16]; while the surgeon's time is precious and easy to cause complaints. Optimizing the cleaning management in the operating room, improving the effective use of the operating room, reducing the working hours and labor intensity of the surgical medical staff, and accelerating the turnover of surgical patients are a win-win reform of medical treatment and patients.

\section{Acknowledgements}

The authors declare no conflict of interest.

\section{References}

[1] Liang Z, Changxiao J, Jun W et al. Research on Methods to Improve Operating Room Efficiency. Chinese hospital 2008: $50-4$.

[2] Li Y, Lijuan L, Xuechun L et al. Application and effect of Delphi method in improving operating room utilization. Contemporary Nurse (Academic Edition) 2014: 180-2.

[3] Zhihong T, Lijun L, Yun Y, Jichun L. Clinical application of improved nursing management measures for serial surgery. Contemporary Nurse (Last Issue) 2015: 79-80.

[4] Jianping G, Xiujuan Z. Implement standardized management of clean operating room to effectively prevent hospital infection. Chinese Journal of Nosocomiology 2012: 186-7.

[5] Li Liuyi ZL, Yao Chushui, etc. Technical specifications for disinfection of medical institutions. 2012.

[6] Jianqing L. Study on Improving Process Management and Operating Efficiency of Operating Room. Journal of Clinical Nursing 2011: 57-8.

[7] houchan C, Hongxia D, Xinlian L et al. Research on Application of Time Management in Optimizing Operation Process of Clean Operating Room. Journal of Nursing 2011.

[8] Juzhen L, Xiaohua C. The role of using root cause analysis to shorten the time to send patients to the table. Modern hospital 2015: $116-7+9$.

[9] Chunhong G. Hidden dangers of preventive surgery and preventive measures. Nursing Practice and Research 2012; 009: 145-6.

[10] Sirui L, Yaojun X, Jing L. Strengthen the management of the operating room and improve the efficiency of the operating room. Chinese hospital 2008: 77-8.

[11] huaying X. Management of cleaners in clean operating room. Chinese Journal of Nosocomiology 2011; 000: 4048.

[12] Xiangchun L, Xiangli L, Yaping H. New progress in training management of clean room operating cleaners. China Practical Medicine 2012; 7: 270-1.

[13] Chernov M, Pullockaran J, Vick A et al. Integrated Practice Improvement Solutions-Practical Steps to Operating Room Management. Journal of investigative surgery: the official journal of the Academy of Surgical Research 2016; 29: 316-21.

[14] Lin C. Application of quality control circle in shortening the management time of laparoscopic surgery. Nursing Practice and Research 2014; 2. 
[15] Wachtel RE, Dexter F. Tactical increases in operating room block time for capacity planning should not be based on utilization. Anesthesia and analgesia 2008; 106: 215-26, table of contents.
[16] Jing W. Hidden safety hazards and protective measures in serial operation. Huaihai Medicine 2011: 83-4. 